sondere die dort definierten altersmäßigen Begrenzungen nicht. Nur Beihilfeberechtigte und Patienten in bestimmten PKV-Tarifen müssen warten, bis sie 35 bzw. 45 Jahre alt sind, bevor sie die Untersuchungen bezahlt bekommen.

\section{MMW-KOMMENTAR}

Gemäß der Empfehlung des Ausschusses Gebührenordnung der Bundesärztekammer kann die Nr. 29 mit dem Hautkrebsscreening per Auflichtmikroskopie nach Nr. 750 kombiniert werden. Der zeitliche Mehraufwand für die kombinierte Beratungsleistung kann über einen erhöhten Steigerungssatz bei der Nr. 29 berechnet werden.

Von der Abrechnung ausgeschlossen sind neben der Nr. 28 die Nrn. 1, 3, 5, 6, 7, 8, 11, 3500, 3511, 3650 und 3652. Neben der Nr. 29 sind die Nrn. 1, 3, 5, 6, 7 und 8 nicht möglich. Wei- tere Leistungen, etwa Laboruntersuchungen oder technische Leistungen wie EKG, Lungenfunktion oder Sonografien, sind nur dann abrechenbar, wenn es einen kurativen Anlass gibt-ggf. auch einen Verdachtsfall. Das gilt auch z. B. für die eingehende neurologische Untersuchung nach Nr. 800, wenn sich aus dem präventiven Untersuchungsbefund ein weiterer neurologischer Untersuchungsanlass ergibt.

\title{
Der Patient will seine Unterlagen - was nun?
}

— Laut $₫ 630$ g Abs. 1 BGB darf jeder Patient die sofortige Einsicht in die eigene Patientenakte fordern. Nur erhebliche therapeutische Gründe - etwa psychiatrische Erkrankungen - oder sonstige erhebliche Rechte Dritter können dem entgegenstehen. Auch das ärztliche Berufsrecht verpflichtet den Arzt in $\$ 10$ Abs. 2 der Musterberufsordnung, seinen Patienten Einsicht in die objektiven Teile der Krankenakte zu gewähren.

Der Arzt steht somit in der Pflicht, seine Patienten auf Wunsch schnellstmöglich die komplette Original-Akte einsehen zu lassen, Kopien anzufertigen, oder eine Auskunft über einzelne $\mathrm{Ab}$ schnitte der Akte zu gewähren. Der Patient hat dagegen kein Recht auf die Aushändigung des Originals. Lediglich Röntgenbilder, die zur Weiterbehandlung notwendig sind, kann er nach $₫ 28$ Abs. 8 der Röntgenverordnung im Original verlangen.

Für die Anfertigung von Kopien kann vorab eine Kostenerstattung verlangt werden. Ein Recht auf das Zusenden von Kopien hat der Patient nicht. Umgekehrt kann der Arzt sich mit einer solchen Zusendung nicht von der Pflicht befreien, Einsicht in die Original-Akte zu gewähren.

Bei einem Arztwechsel entscheidet der Patient selbst, welche Informationen der neue Arzt erhält. Er kann dem vorherigen
Arzt erlauben, dem neuen Arzt seine Akte zu leihen, damit dieser sich einen Überblick verschaffen kann. Außerdem kann der Patient Kopien der Akte verlangen, um diese selbst auszuhändigen.

Der neue Arzt hingegen hat selbst kein Recht darauf, die Patientenakte ohne eine schriftliche Einverständniserklärung und somit Entbindung von der Schweigepflicht einzusehen. Auch das Praxispersonal ist ohne diese Erklärung nicht befugt, anderen Ärzten Auskunft zu geben. Erlaubt der Patient, dass die komplette Originalakte an einen neuen Arzt gegeben wird, so darf dies nur geschehen, wenn die Behandlung bereits seit zehn Jahren abgeschlossen ist denn der ursprüngliche Arzt muss die Aufbewahrungsfrist einhalten und darf in dieser Zeit keine Originalakte abgeben, zumal diese Akte als Eigentum des Arztes gilt.

\section{MMW-KOMMENTAR}

Zur Möglichkeit einer Kostenerstattung von erbetenen Kopien und der da-

für in der Regel anzusetzenden Kosten gibt es die unterschiedlichsten Stellungnahmen. Keineswegs können sich Ärzte an den sonst bei Ämtern oder Behörden üblichen Sätzen orientieren. In Analogie zum Justizvergütungs- und Entschädigungsgesetz (JVEG) kann man aber für bis zu 50 Seiten 0,50 Euro je Seite vom Patienten verlangen, für jede weitere Seiten 0,15 Euro. Wenn der Patient eine $C D$ erhält, um diese einem weiteren behandelnden Arzt vorübergehend zu überlassen, kann keine Rechnung gestellt werden. Der mitbehandelnde Arzt muss allerdings die $C D$ zurückschicken. Anders ist es, wenn der Patient die CD für die eigene Dokumentation erbittet. Auch hier ist in Analogie zum JVEG ein Betrag von 1,50 Euro je Datei in Rechnung zu stellen. Werden mehrere Dateien, z.B. mehrere Sonografie- oder Röntgenuntersuchungen, auf eine CD übertragen, beträgt der zulässige Höchstsatz 5 Euro. Bei Privatpatienten kann für das Überlassen einer $C D$ auf Wunsch eine Abrechnungsempfehlung der Bundesärztekammer herangezogen werden [Dtsch Arztebl. 2012;109:A987]. Auch hier werden 5 Euro genannt.

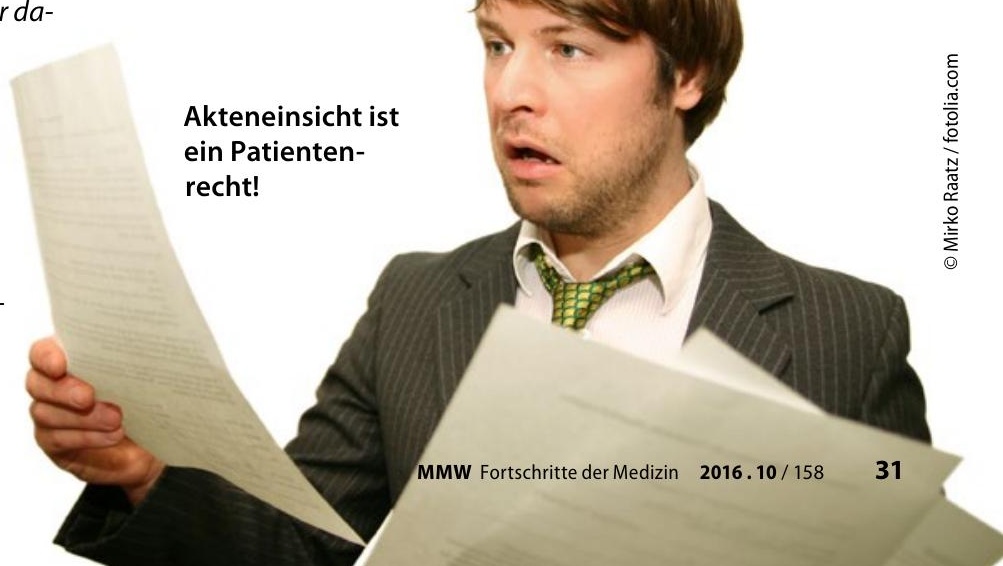

